\section{Primera colecistectomía laparoscópica en el Perú}

\author{
First laparoscopic cholecystectomy in Peru
}

\author{
Mario A. Del Castillo-Yrigoyen'
}

Desde el siglo $X$ en que Abulcasis realizo la primera visión endoscópica del cérvix uterino, muchos inventos sucedieron a lo largo de los años con el sueño de visualizar los órganos internos del ser humano, estudiarlos y mejorar el diagnóstico de algunas enfermedades, Philip Bozzini realiza la primera endoscopia de la vejiga en 1805, Dimitrij Ott y Georg Kelling en 1901 inventan el primer laparoscopio y Hans Jacobeus en 1901 realiza la primera laparoscopia en humanos, posteriormente Carl Fervers en 1933 mejora la técnica laparoscópica con la utilización de $\mathrm{CO}_{2}$ para el neumoperitoneo y tener mejor visión de los órganos intraabdominales y como ginecólogo realiza las primera liberación de adherencias pélvicas por infertilidad, es así como los ginecólogos se adelantaron en la laparoscopia no solo diagnostica sino operatoria, en 1935 PF Bosch realiza la primera ligadura tubaria por laparoscopia. Kurt Semm en 1962 utiliza por primera vez el insuflador automático, en 1973 la endocoagulación por laparoscopia, en 1982 fue el primero en utilizar la endocámara y realizar la primera apendicetomía laparoscópica.

En el Perú, la historia de la laparoscopia data del año 1957, iniciada por los gastroenterólogos como el Dr. Hernán Espejo Romero en 1957 en el Hospital HERM, el Dr. Rolando Figueroa Barrios en 1962 en el Hospital Dos de Mayo y el Dr. Ernesto Castillo Lindley del Hospital de Policía, posteriormente muchos ginecólogos de los hospitales Cayetano Heredia y Arzobispo Loayza realizaron procedimientos diagnósticos y operatorios bajo visión directa.

I Cirujano general. Ex jefe del Departamento de Cirugía del Hospital Nacional Cayetano Heredia. Profesor principal, Facultad de Medicina, Universidad Peruana Cayetano Heredia, Lima, Perú.
Del Castillo-Yrigoyen M. Primera colecistectomía laparoscópica en el Perú. 2021;34(I): 45-47.

https://doi.org/10.36393/spmi.v34i1.585

La colecistectomía laparoscópica fue la operación emblemática en el avance de esta técnica operatoria, desde sus inicios con Erich Mühe en 1985, siguiendo en Francia con Francois Dubois y Philippe Mouret en 1987 para posteriormente en EEUU, Mc Kernan, Redick y Olsen realizaran las primeras colecistectomías en 1989. En Latino América no fuimos muy ajenos a esta nueva alternativa quirúrgica para operaciones de la vesícula, Thomas Szego en Brasil en Julio de 1990, Alfredo Sepúlveda y Carlos Lizana en agosto del mismo año y en el Perú, Mario Del Catillo y Manuel Rodríguez en octubre de 1990.

Este impulso en la cirugía nace del entusiasmo del Dr. Roger Guerra García, Rector de la Universidad Peruana Cayetano Heredia, con quien en esos años difíciles de la medicina, con pocos recursos tecnológicos y escasas posibilidades de avances sustanciales en mi especialidad de Cirugía General, acudí al Rectorado con el fin de proponerle que se podían gestionar donaciones por parte de los exalumnos de la Universidad en EEUU, donde forman una Asociación, el Peruvian American Endowment, muchos de ellos compañeros de promoción de la UPCH y amigos de otras promociones. Después de realizar algunos contactos y ver la posibilidad de donaciones por parte de algunos hospitales donde trabajan estos compañeros de universidad, se tomó la decisión de que viajara a Chicago donde el Dr. Edgar Del Castillo, exalumno de la UPCH y Ginecólogo. Con su entusiasmo el Dr. Guerra me converso que además de las donaciones, vea los avances en la cirugía laparoscópica. Con esta idea, estuve primero en el South Suburban Hospital, donde trabajaba el Dr. Del Castillo, quien ya hacía mucha laparoscopia ginecológica. Posteriormente, viajo a Detroit donde otro exalumno de la UPCH, el Dr. Germán Barbe, cirujano general, quien trabaja en el St. John Hospital, me permitió asistir a un sin 
número de operaciones de colecistectomía laparoscópica. A mi regreso al Perú en agosto de 1990, converse con un cirujano joven, recién egresado de la residencia del Hospital Cayetano Heredia, el Dr. Manuel Rodríguez, sobre las grandes ventajas y posibilidades de realizar esta nueva técnica en nuestro país y comenzamos a trabajar día a día, tratando de conseguir todos los equipos necesarios para este tipo de cirugía, el instrumental adecuado y de realizar prácticas en animales en el laboratorio de Técnica Operatoria y Cirugía Experimental de la UPCH.

El comienzo no fue nada fácil, la fuerte oposición por algunos jefes y colegas de nuestro hospital, dificultaban aún más el operar con esta nueva técnica operatoria. Sin embargo; el 23 de octubre de 1990, realizamos las dos primeras colecistectomías laparoscópicas Figuras 1 y 2). No decaímos en nuestra decisión de usar esta nueva técnica, para la cual estábamos capacitados y preparados, realizando nuestras siguientes cirugías en una clínica privada. En el medio local, también teníamos una fuerte oposición por parte de los cirujanos más experimentados, sobre todo que pertenecían a sociedades médicas como la Academia Peruana de Cirugía y la Sociedad de Cirujanos Generales. Pero nuestro entusiasmo nunca decayó, asistimos a todos los eventos posibles con el fin de presentar esta nueva alternativa en la cirugía, entusiasmando a otros cirujanos jóvenes que posteriormente desarrollarían este tipo de cirugía con gran éxito. Y, así, realizamos la primera publicación: Del Castillo Yrigoyen M, Rodríguez Castro M. Colecistectomía laparoscópica en el Perú. Reporte preliminar de los primeros casos. Revista Médica Herediana. 1991;2(1): 41-42.

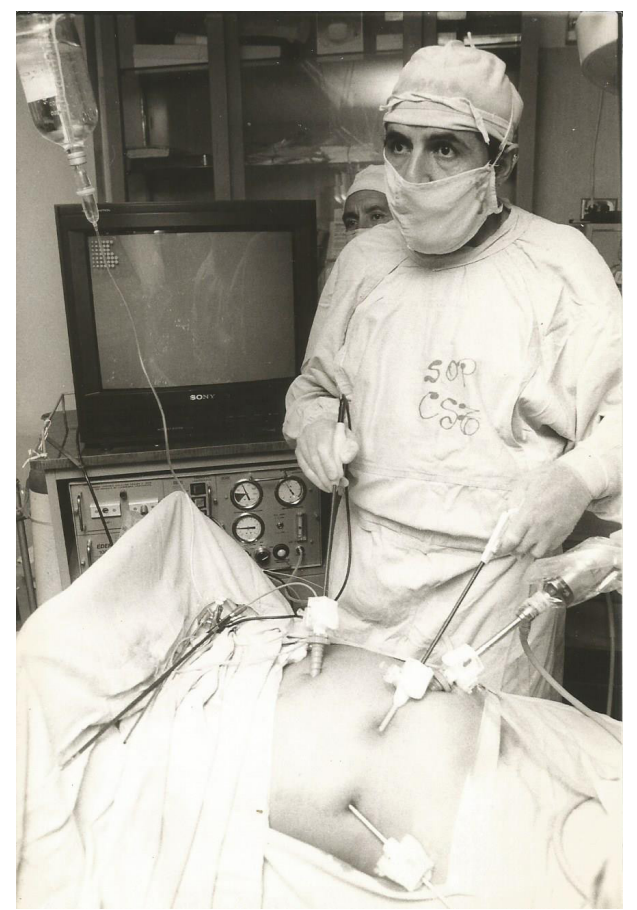

Figura I. Dr. Mario Del Castillo Yrigoyen
También fuimos fundadores en 1994 de los primeros cursos regulares de Cirugía Laparoscópica en el Centro de Entrenamiento de Cirugía Laparoscópica (CECIL) que realizamos en la UPCH, como un medio de contribución y difusión de esta cirugía, con prácticas en cajas de entrenamiento, animales y demostración en pacientes del Hospital Cayetano Heredia. Tuvimos el honor de enseñar a nuestros distinguidos profesores como el Dr. Víctor Baracco Gandolfo, jefe del Departamento de Cirugía por muchos años y el Dr. Eduardo Barboza Besada, jefe del Departamento de Cirugía de la UPCH, ambos referentes en mi formación como cirujano general.

Con el correr del tiempo, nuevos grupos de cirujanos completaron su entrenamiento y comenzaron a operar en diferentes instituciones públicas y privadas. En 1997 nos reunimos 13 cirujanos y acordamos fundar la Sociedad de Cirugía Laparoscópica, de la que fui presidente por dos periodos.

Durante nuestra gestión en la Jefatura del Departamento de Cirugía del $\mathrm{HNCH}$, implementamos en el 2015 la Unidad de Cirugía Mayor Ambulatoria (UCMA), en la que se operaban a pacientes seleccionados bajo una directiva estricta de colecistectomía Laparoscópica en forma ambulatoria, contribuyendo de esta manera a la gran población de pacientes con enfermedad de la vesícula que se operaban y salían de alta el mismo día, sin ocupar una cama hospitalaria y disminuyendo los costos en forma importante.

Cuando comenzamos esta cirugía, llamada hoy video endoscopia quirúrgica, no pensamos que estaríamos dando inicio a una era importante en la Cirugía moderna en el Perú, trascendiendo y avanzando en la gran mayoría de especialidades quirúrgicas, desde la misma cirugía

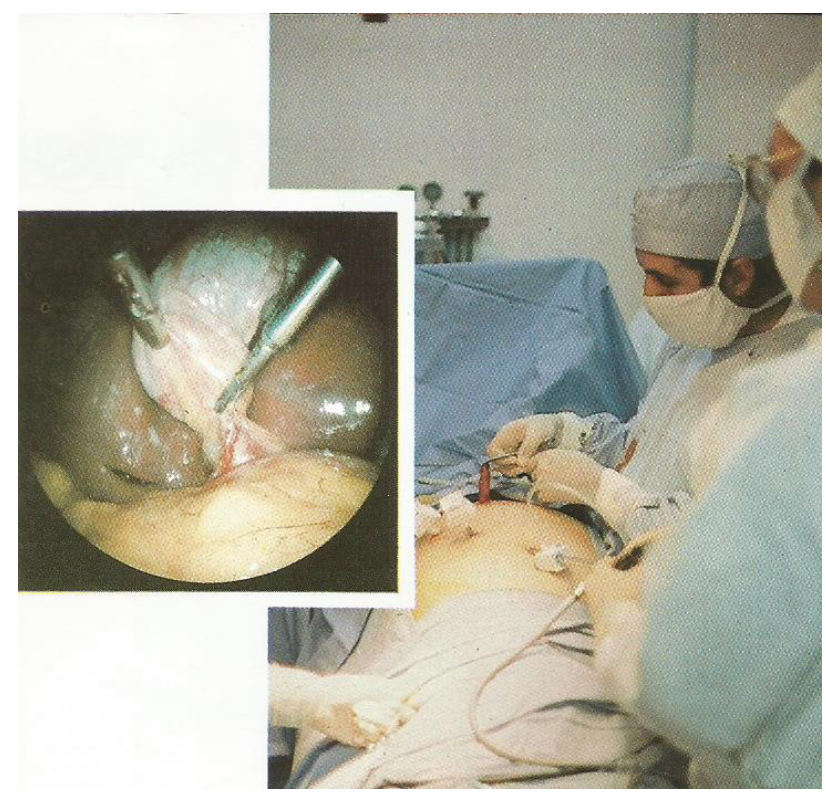

Figura 2. Primera colecistectomía laparoscópica, 23 de octubre de 1990. 
general o digestiva, con operaciones tan complejas como son la colectomía, operaciones por Cáncer hasta la duodenopancreatectomia. En otras especialidades como la neurocirugía, traumatología, urología, cardiocirugia, etc. cada una con un equipo e instrumental que avanza día a día. Este año, después de 30 años de haber sido los pioneros de la cirugía laparoscópica en el Perú, quiero expresar mi agradecimiento a la UPCH en la persona del Dr. Roger Guerra García quien nos impulsó y apoyo en este proyecto, al Hospital Nacional Cayetano Heredia que nos dio la oportunidad de operar por primera vez una colecistectomía laparoscópica, a nuestro profesores doctores Víctor Baracco Gandolfo y Dr. Eduardo Barboza Besada que sin su ejemplo, orientación y enseñanzas no hubiera podido alcanzar los éxitos en mi carrera como cirujano general.

\section{CORRESPONDENCIA}

Mario Del CastilloYrigoyen

mario.del.castillo@upch.pe

Fecha de recepción: 3|-08-2020.

Fecha de aceptación: 10-0I-202I. 